\title{
New Tools for the Study of Deformed and Heat-Treated Materials via Electron Backscatter Diffraction
}

\author{
Travis M. Rampton ${ }^{1}$, Matthew M. Nowell ${ }^{2}$, Stuart I. Wright ${ }^{2}$ \\ ${ }^{1}$ EDAX, A Division of Ametek, Materials Analysis Division, Mahwah, NJ USA. \\ 2. EDAX, A Division of Ametek, Materials Analysis Division, Draper, UT USA.
}

The commercialization of electron backscatter diffraction (EBSD) systems over 25 years ago has allowed for the routine analysis of crystalline materials ranging from metals to ceramics and composites. Since the first EBSD systems were used they have been used by a variety of research fields to extract an increasing amount of information, but no area of study has been as critical as the ability to understand and quantify deformation in materials. Increased insights into deformed materials are critical to improving processes such as rolling, extrusion, and heating. Traditionally quantifying deformation has been tied to measures of local misorientation such as grain average misorientation or kernel average misorientation $[1,2]$. However, no universal measure has been employed to correlate with deformation; thus, it is advantageous to develop new methods for measuring and quantifying deformation until such an all-encompassing technique becomes available.

This study will present two new advances to EBSD which are available in EDAX's TEAM and OIM Analysis software packages that can be applied to the study deformed materials. The first tool is the pattern region of interest analysis system (PRIAS) which as the name describes uses selected areas of an EBSD pattern or the EBSD detector to extract a myriad of information including, but not limited to, orientation gradients caused by deformation. This type of tool is able to be applied either before, during, or after an EBSD scan has taken place. When used prior to a scan the PRIAS is particularly powerful because it is able to operate much faster than standard EBSD by binning the detector image to much smaller than can be used for standard orientation. The second new tool that will be explored with this study is the grain reference orientation deviation (GROD) - axis map. Instead of considering the angle of a local misorientation this metric looks at the axis about which the local misorientation occurs relative to some reference orientation, usually the average orientation of each grain. Such a measure has proven useful in seeing localized "packets" of deformation. Additionally, GROD - axis show the crystallographic direction of rotation which could potentially be tied to a set of slip systems thus allowing for correlation with a Taylor model.

The usefulness of these tools will be demonstrated on undeformed materials as they progress through a series of deformation steps (Fig. 1) as well as some deformed materials as they are heat treated to remove deformation (Fig. 2). 


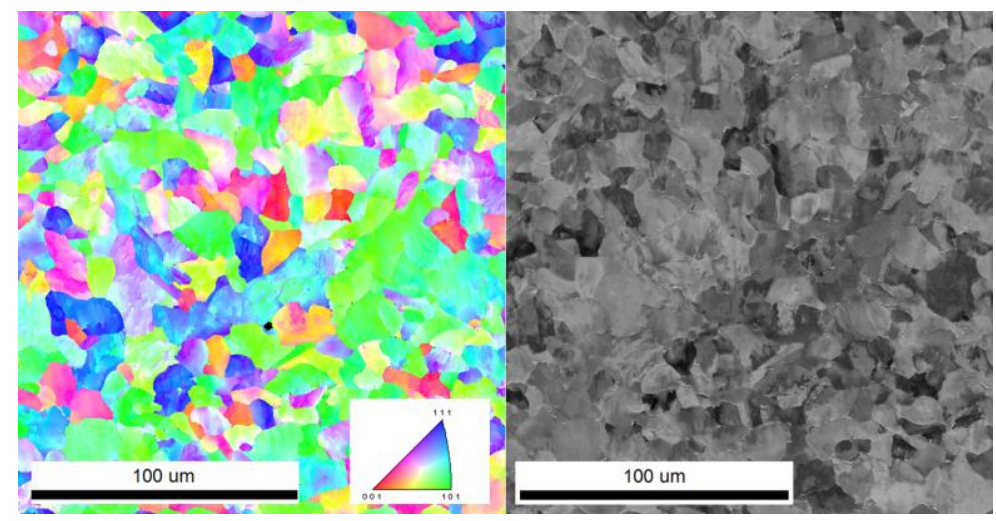

Figure 1. (Left) Inverse pole figure (IPF) map and (Right) PRIAS map of deformed steel structure

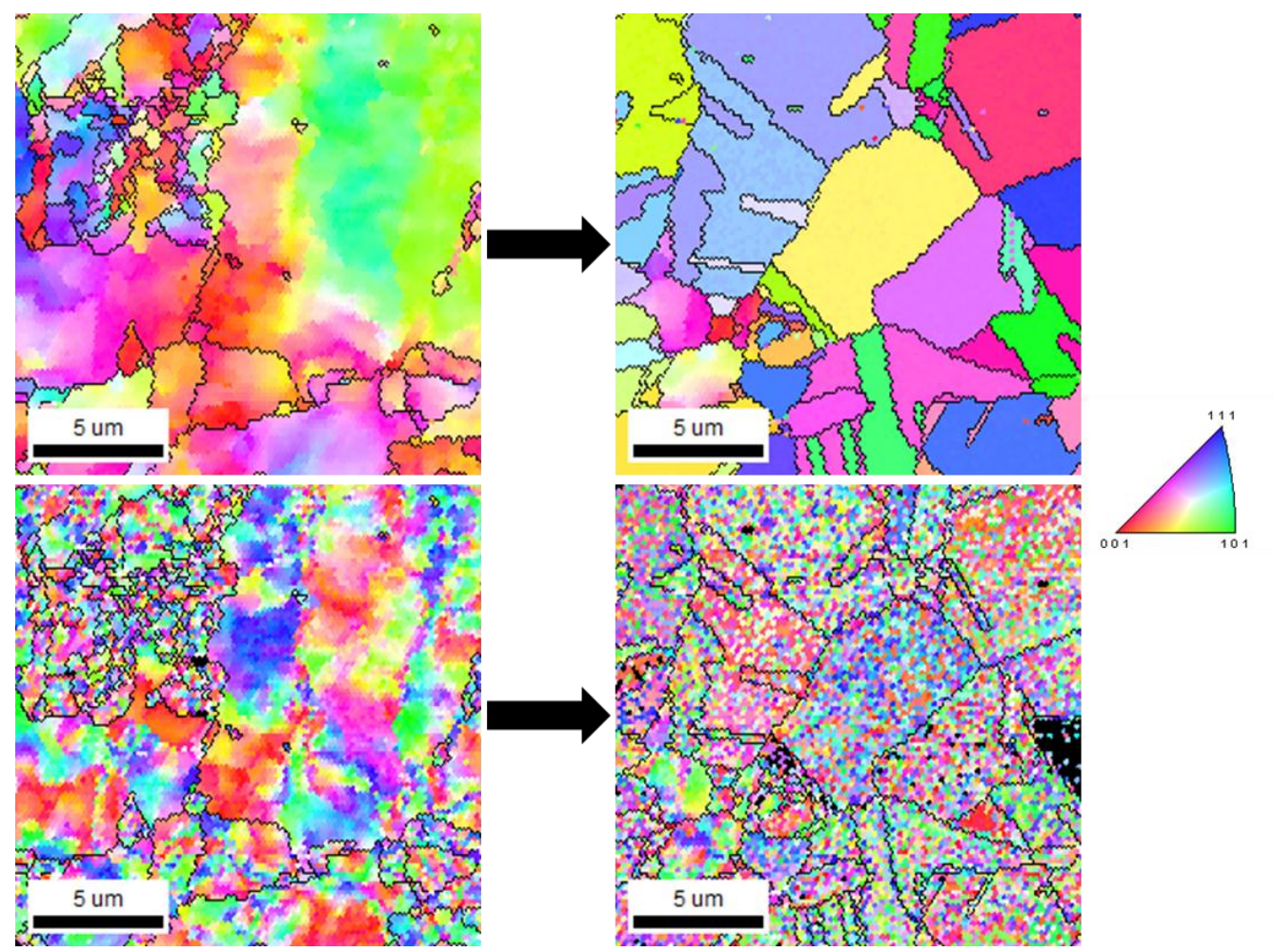

Figure 2. (Top Left) IPF map and (Bottom Left) GROD axis map of initially deformed copper microstructure. (Top Right) IPF map and (Bottom Right) GROD axis map of deformed copper microstructure after final step of heat treatment. The deformation is clearly seen in the GROD axis map of the initial microstructure as opposed to the final microstructure where the local misorientations are below the threshold of EBSD and thus appear as noise in the GROD axis map.

\section{References:}

[1] R. Yoda, T. Yokomaku and N. Tsuji, Materials Characterization 61 (2010), p.913-922

[2] S. Wright, M. Nowell and D. Field, Microsc. Microanal. 17 (2011), p. 316-329 